\title{
Aprendizado de Lógica Matemática: Avaliação de Apps para Tabela Verdade e Recomendação de Uso
}

\author{
Bruna de Abreu Dias ${ }^{1}$, Marina Braun Otokovieski ${ }^{1}$, \\ Alice Fonseca Finger ${ }^{1}$, João Pablo Silva da Silva ${ }^{1}$, Miguel Ecar ${ }^{1}$ \\ ${ }^{1}$ Laboratory of Intelligent Software Engineering (LabISE) \\ Universidade Federal do Pampa (UNIPAMPA) - Campus Alegrete \\ Av. Tiarajú, 810, Ibirapuitã - Alegrete, RS - Brasil \\ \{brunadias.aluno, marinaotokovieski.aluno\}@unipampa.edu.br \\ \{alicefinger, joaosilva\}@unipampa.edu.br, miguel@ecarsm.com
}

\begin{abstract}
Mathematical bias disciplines are known for the high rates of dropout and retention in Computing related courses. Combining teaching strategies and technology have contributed to reducing the negative rates. Based on this, several educational tools have been created in order to make teaching and learning more attractive. The present work aims to evaluate two apps usability Truth Table building, in order to point out possible improvements, as well as, to encourage its use in learning Mathematical Logic. The results, based on the System Usability Scale (SUS) metric, show that the Tablas de Verdad PRO app has a good degree of usability, while The Logic Calculator app has not reached the minimum acceptable value.
\end{abstract}

Resumo. Disciplinas com viés matemático são conhecidas pelos altos índices de evasão e retenção em cursos na área da Computação. Estratégias de ensino aliadas à Tecnologias Digitais da Informação e Comunicação (TDICs) vem contribuindo para diminuir os índices, uma vez que diferentes ferramentas educacionais são criadas com o intuito de tornar o ensino mais atrativo. $O$ presente trabalho objetiva avaliar a usabilidade de dois aplicativos para construção de Tabela Verdade (TV) a fim de apontar possiveis melhorias, bem como incentivar seu uso nas disciplinas de SUS Os resultados, a partir da métrica SUS mostram que o aplicativo Tablas de Verdad PRO possui um bom grau de usabilidade, enquanto o aplicativo The Logic Calculator não atingiu o valor mínimo aceitável.

\section{Introdução}

Cursos de graduação na área de Computação são conhecidos pelos altos índices de evasão e retenção [SBC 2018]. Aliado a isso, a dificuldade com conteúdos matemáticos e teóricos contribuem diretamente para a alta desses números. Entender os conceitos matemáticos é fundamental para um profissional da área, uma vez que a Ciência da Computação depende da Matemática para obter um vocabulário preciso, uma notação poderosa, abstrações úteis e um raciocínio rigoroso [Gersting 2001].

As TDICs podem ser vantajosas aliadas no processo de ensino-aprendizagem [Jurgina et al. 2020, Souto and Silva 2017], uma vez que, no atual momento em que se 
encontra o ensino, os estudantes e professores estão cada vez mais conectados e as ferramentas tecnológicas fazem parte da rotina de todos. Trabalhos mostram que utilizar jogos educacionais, por exemplo, proporcionam aos estudantes e profissionais um ambiente interativo e dinâmico provocando a motivação do discente pelo conteúdo da disciplina [Souza and França 2016]. Além disso, objetos de aprendizagem estão sendo criados e utilizados por meio da $m$-learning, com smartphones para o ensino [Andrade et al. 2017], porém, é fundamental que se desenvolvam mecanismos de avaliação desses objetos, no intuito de que eles sejam uma fonte de conhecimento e prática no ensino-aprendizagem.

Entre as disciplinas matemáticas para Computação, destaca-se a Lógica Matemática (LM), na qual o objetivo vai além de apenas estimular o raciocínio lógico. Ela introduz uma nova linguagem que pode ser usada como linguagem de programação e pode apresentar formas de abstração servindo de ferramenta a ser usada na definição formal de conceitos computacionais. Para auxiliar na compreensão dos seus conceitos, foi realizada uma revisão sistemática que buscava por aplicativos para dispositivos móveis que servissem de auxílio no ensino-aprendizado de LM] [Dias and Finger 2020]. Entre as 19 aplicações encontradas, 8 tinham foco voltado para construção de TV] porém elas carecem de avaliações da usabilidade, o que além de medir a qualidade, pode também influenciar na escolha para uso em sala de aula.

A Engenharia de Software estabelece uma série de práticas orientadas ao controle, garantia e gerenciamento da qualidade de software. Atrelado a isso, normas e padrões são criados no intuito de elencar um conjunto de características que devem ser verificadas em um software para que ele seja considerado um software de qualidade, bem como métricas usadas na sua avaliação [Pressman 2011, Sommerville 2011]. Ainda não existem normas que especifiquem critérios de qualidade para aplicativos, mas alguns trabalhos na literatura recomendam critérios de avaliação no contexto de aplicativos para ensinoaprendizagem, sendo um deles a usabilidade, a qual avalia a facilidade de uso e aprendizagem [Andrade et al. 2017].

A pesquisa realizada obteve resultados quantitativos e qualitativos e seu objetivo é avaliar a usabilidade de dois aplicativos de TV] com o foco principal de incentivar seu uso como objeto de aprendizagem na disciplina de SUS, a partir da visão e avaliação dos alunos envolvidos no teste, além de apontar possíveis melhorias. Para isso, foi realizado um teste de usabilidade, o qual constitui em um procedimento com passos definidos para que seja possível colher dados da interação que ocorre entre usuário e produto [Fernandez et al. 2011].

O trabalho está organizado da seguinte maneira: a Seção 2 descreve as principais características dos aplicativos para TV, na Seção 3 apresenta-se a metodologia utilizada na construção do trabalho; a Seção 4 mostra os resultados do teste de usabilidade, algumas sugestões de melhorias e as ameças à validade do teste e a Seção 5 resume as principais considerações do trabalho.

\section{Aplicativos para Tabela Verdade}

No trabalho desenvolvido por [Dias and Finger 2020], foi realizada uma revisão sistemática no intuito de investigar aplicativos disponíveis na Google Play Store que auxiliem no ensino-aprendizado de LM. Entre eles, foram encontradas aplicações para TV que utilizam a semântica de Operador Lógico (OL) para classificar Fórmula Bem Formada (FBF) 
e validar argumentos. A sintaxe das fórmulas lógicas inclui o uso de letras do alfabeto e OL, podendo também utilizar parênteses, se necessário [de Souza 2008]. A partir de uma análise de características e funções dos aplicativos encontrados, Tablas de Verdad PRO e The Logic Calculator obtiveram maior destaque após avaliação de suas funções, conforme resume a Tabela 1 .

Tabela 1. Características e funções presentes nos aplicativos.

\begin{tabular}{|c|c|c|c|c|c|c|c|}
\hline Identificador & Aplicativos & FBF & Argumentos & Parênteses & Alfabeto & OL Básico & Outros \\
\hline App-A & Tablas de Verdad PRO & $\checkmark$ & & $\checkmark$ & 26 & $\checkmark$ & $\checkmark$ \\
\hline App-B & The Logic Calculator & $\checkmark$ & $\checkmark$ & $\checkmark$ & 6 & $\checkmark$ & \\
\hline
\end{tabular}

A Tabela 1 apresenta em sua primeira coluna o identificador atribuído para cada aplicativo, com seu nome definido na segunda coluna, no intuito de resumir explicações posteriores. Em seguida, as demais colunas apresentam indicações sobre os aplicativos que possuem disponibilidade para: classificação de uma $\overline{\mathrm{FBF}}$; classificação de argumentos; uso de parênteses. Ainda, a informação da quantidade de letras disponíveis em seus alfabetos e a identificação da disponibilidade dos OL básicos (negação, conjunção, disjunção, condicional e bicondicional) e outros OL (XOR, NAND, NOR).

O aplicativo App-A atualmente está disponível na Google Play Store ${ }^{1}$ em 3 versões: inglês, espanhol e PRO. Porém, desde a realização da revisão sistemática, a única diferença observada entre elas é que a versão PRO não contém anúncios. Nas 3 versões é possível modificar o idioma de inglês para espanhol e vice-versa. Notou-se também que, em versões anteriores, os aplicativos continham explicações sobre o cálculo da TV]e agora não há essa função. Durante a revisão sistemática, a versão PRO não estava disponível, porém, para realização do teste constatou-se as diferenças mencionadas anteriormente e optou-se pela utilização da versão PRO por não conter anúncios que poderiam prolongar e dificultar o andamento do teste. Destaca-se que, até o momento do teste, as 3 versões eram gratuitas. Em relação à outras características do App-A, destaca-se que sua última atualização ocorreu em 2021, e está atualmente na versão atual 2.0. Possui um tamanho de $19 \mathrm{MB}$, tem mais de mil instalações, seu idioma principal é o espanhol e dispõe de uso offline.

O aplicativo App-B se encontra na Google Play Store ${ }^{2}$ e, diferente do App-A, não foram observadas diferenças desde a revisão sistemática [Dias and Finger 2020]. Sua última atualização ocorreu em 2019 e atualmente está na versão 1.1. Seu tamanho varia conforme o dispositivo, contém mais de cinco mil instalações e seu idioma é o inglês.

Como pode ser visualizado na Figura 1, os aplicativos possuem uma interface simples, ambos disponibilizam um alfabeto, porém com tamanhos distintos. $\mathrm{O}$ aplicativo App-A pode ser visto na Figura 1a e App-B na Figura 1b. É possível encontrar os OL básicos com diferentes representações, como a conjunção que no App-B representada por \& e no App-A sinalizada com o símbolo $\wedge$. Em seus teclados, as aplicações contêm parênteses e duas formas de apagar a fórmula escrita, uma exclui a fórmula completa no App-A representado pelo ícone de uma lixeira e no App-B pela letra C - e a outra exclui um item por vez, nas duas aplicações representadas por uma seta que contém a

\footnotetext{
${ }^{1}$ Tablas de Verdad PRO. Acesso pela Google Play Store

${ }^{2}$ The Logic Calculator. Acesso pela Google Play Store
} 




(a) App-A: Tablas de Verdad PRO

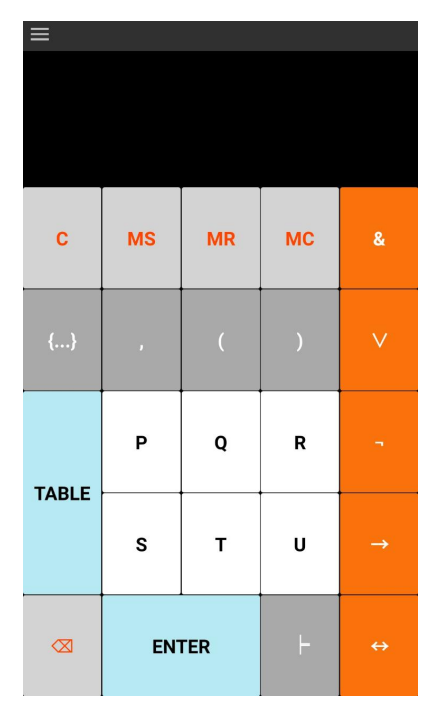

(b) App-B: The Logic Calculator

Figura 1. Tela inicial dos aplicativos.

letra xis. Destaca-se que os aplicativos se diferem na forma de apresentar o resultado da tabela referente a fórmula lógica, pois no App-A essa opção é representada pelo sinal de igualdade (=) e no App-B é possível visualizá-lo clicando em "Table", e ao clicar no botão "Enter" o retorno obtido é a classificação ou validação da fórmula inserida.

\section{Metodologia}

Após a realização da revisão sistemática com aplicativos, bem como o comparativo entre as funções observadas, destacou-se o App-B (The Logic Calculator) pois é o único capaz de construir uma TV] para argumentos [Dias and Finger 2020]. Como o objetivo deste trabalho é a realização de um teste de usabilidade e comparativo entre os aplicativos, o App-A (Tablas de Verdad PRO), também presente na revisão sistemática, foi selecionado. Anteriormente o aplicativo não foi evidenciado por não ser tão completo quanto o App-B, mas foi escolhido para o teste devido ao seu extenso alfabeto e possibilidade de visualizar o passo a passo dos resultados. Em seguida, analisando os aplicativos e suas funcionalidades, criaram-se 4 cenários $3^{3}$ de teste, distribuídos em 2 grupos e com roteiros distintos, ou seja, fazendo o uso de funções e OL diferentes. Salienta-se que o objetivo do teste é que o usuário siga todos os passos de maneira correta e possa ter uma completa interação com o aplicativo. Por isso os passos são descritos de maneira a induzir em qual lugar clicar para realizar a tarefa. A Tabela 2 sintetiza as características de cada cenário.

Os cenários estão dispostos no intuito de destacar as principais diferenças entre eles. A primeira coluna contém um código correspondente aos grupos e aos cenários em cada grupo (exemplo: grupo I com seu cenário I gera o código I.I), já na segunda coluna, é identificado o aplicativo utilizado. Em seguida, apresenta-se: a quantidade de OL testados; a possibilidade de visualizar os resultados; a classificação do tipo de TV; a verificação da validade do argumento; a possibilidade de visualizar o passo a passo da construção da TV; a funcionalidade de realizar uma cópia da TV criada e a alternativa de

\footnotetext{
${ }^{3}$ https://bit.ly/TesteCenarios
} 
Tabela 2. Funções verificadas nos cenários.

\begin{tabular}{|c|c|c|c|c|c|c|c|c|}
\hline $\begin{array}{c}\text { Grupo/ } \\
\text { Cenário }\end{array}$ & App & OL & $\begin{array}{c}\text { Visualizar } \\
\text { resultado }\end{array}$ & $\begin{array}{c}\text { Verificar } \\
\text { classificação }\end{array}$ & $\begin{array}{c}\text { Verificar } \\
\text { validade }\end{array}$ & $\begin{array}{c}\text { Visualizar } \\
\text { passo a passo }\end{array}$ & Copiar Tabela & $\begin{array}{c}\text { Mudar valores } \\
\text { da TV }\end{array}$ \\
\hline I.I & App-A & 4 & $\checkmark$ & $\checkmark$ & & $\checkmark$ & & $\checkmark$ \\
\hline I.II & App-B & 5 & & $\checkmark$ & & & & \\
\hline II.I & App-A & 3 & $\checkmark$ & $\checkmark$ & & $\checkmark$ & & \\
\hline II.II & App-B & 4 & $\checkmark$ & & $\checkmark$ & & $\checkmark$ & \\
\hline
\end{tabular}

alterar os valores das TV.

Os cenários I, referentes aos dois grupos, incluíram o uso de todos os OL básicos, além da funcionalidade de visualizar o resultado da tabela e, consequentemente, sua classificação. Ainda, ambos cenários solicitavam o uso da visualização do passo a passo da TV criada. A diferença entre os grupos estava no fato que, para o cenário do grupo I, antes de realizar os cálculos para geração da TV, era necessário alterar os valores, que inicialmente eram $\mathrm{V}$ ou $\mathrm{F}$, para 0 ou 1.

Os cenários II, de ambos os grupos, também continham todos os OL básicos. Porém, no grupo I, somente a classificação da TV] era visualizada. Já para o grupo II, o cenário abrangia as funções que possibilitavam visualizar o resultado, bem como a validade da forma de argumento. Além disso, era necessário utilizar a função de cópia da TV gerada e enviá-la para o e-mail pertencente ao grupo dos autores do presente artigo. Salienta-se que esse envio por e-mail serviu apenas para que os usuários do teste utilizassem a função de cópia da tabela por completo. Também foi solicitado aos usuários antes do início do teste, que preenchessem um Termo de Consentimento, a fim de permitir a gravação, já que ele ocorreu por meio da plataforma Google Meet.

\subsection{Teste de Usabilidade}

O teste de usabilidade ocorreu no dia 17 março de 2021 com discentes que estavam cursando a disciplina extra de LM. Nela, haviam alunos dos cursos de graduação em Engenharia de Software, Ciência da Computação, bem como outros cursos da Universidade Federal do Pampa (UNIPAMPA), que haviam aprendido o conteúdo sobre $\mathrm{OL}$ e $[\mathrm{TV}] \mathrm{re}$ centemente. Ao início, foram explicadas as condições para que o usuário participasse do teste e ele deveria ter em mãos um smartphone Android que possibilitasse a instalação de ambos aplicativos. Em seguida, foi disponibilizado aos presentes o Termo de Consentimento, no qual obteve-se um total de 17 alunos respondentes. Após, realizou-se um sorteio entre os discentes que haviam assinado o Termo para que a formação do grupos ocorresse de maneira aleatória, deixando assim o grupo I com uma composição de 8 participantes e o grupo II com 9.

Os grupos foram encaminhados para salas separadas na plataforma Google Meet e, em ambas, haviam responsáveis coordenando e auxiliando os participantes. A primeira parte do roteiro foi apresentada e correspondia ao App-A. Assim, conforme os participantes concluíam o cenário, um formulário referente à aplicação foi disponibilizado. O mesmo ocorreu com o cenário para o App-B. Os formulários a serem respondidos pelos participantes, foram criados com base nas questões da SUS. Desenvolvida por [Brooke 1996], a medida aplica dez questões ao usuário, com respostas em uma escala de 1 a 5 , sendo 1 - discordo fortemente e 5 - concordo fortemente, também conhecida 
como Escala Likert [Likert 1932]. A medida SUS foi escolhida por ser bastante utilizada na literatura e se mostrar uma escala de aplicação rápida, simples, eficaz e eficiente para diferentes tipos e complexidades de sistemas [Peres et al. 2013].

\section{Resultados}

Com base na análise dos resultados é possível obter informações que auxiliam na detecção de problemas de usabilidade nos sistemas [Valentim et al. 2014]. A partir dos resultados coletados por meio dos formulários disponibilizados para os alunos dos grupos I e II, realizou-se uma análise utilizando a medida SUS. As dez questões que compõem a SUS estão apresentadas no Quadro 1:

\begin{tabular}{|c|l|c|l|}
\hline \multicolumn{3}{|c|}{ Questões SUS } \\
\hline 1 & $\begin{array}{l}\text { Eu acho que eu gostaria de utilizar este } \\
\text { sistema frequentemente. }\end{array}$ & 6 & Eu achei que tinha muito inconsistência no sistema. \\
\hline 2 & Eu achei o sistema desnecessariamente complexo. & $\begin{array}{l}\text { Eu acho que a maioria das pessoas iriam aprender } \\
\text { facilmente a utilizar esse sistema. }\end{array}$ \\
\hline 3 & Eu achei o sistema fácil de usar. & 8 & Eu achei o sistema muito incômodo de usar. \\
\hline 4 & $\begin{array}{l}\text { Eu acho que eu precisaria da ajuda de um técnico para } \\
\text { eu conseguir utilizar o sistema. }\end{array}$ & 9 & Eu me senti muito confiante em utilizar o sistema. \\
\hline 5 & $\begin{array}{l}\text { Eu acho que as várias funcionalidades do sistemas estão } \\
\text { bem integradas. }\end{array}$ & 10 & $\begin{array}{l}\text { Eu precisei aprender muito antes de utilizar } \\
\text { esse sistema. }\end{array}$ \\
\hline
\end{tabular}

\section{Quadro 1. Questões da escala SUS}

Os resultados alcançados pelo uso do SUS, a partir dos cálculos feitos, permite obter uma visão geral do grau de usabilidade dos sistemas em estudo. No primeiro cálculo, é preciso que as respostas dos usuários a partir da escala Likert em questões ímpares $(1,3,5,7$ e 9) sejam subtraídas de 1 (respostas - 1). Nas questões pares (2, 4, 6, 8 e 10), é subtraído 5 da resposta da escala Likert ( 5 - resposta). Para a pontuação final, os resultados devem ser um a um, multiplicados por 2,5 [Brooke 1996]. Os resultados quantitativos obtidos a partir da escala SUS e das respostas dos participantes coletadas dos formulários, foram adicionados à Tabela 3 . Em função do grupo I conter um participante a menos, a sua última coluna encontra-se sem valor.

Tabela 3. SUS Score: Resultado por participante.

\begin{tabular}{|c|c|c|c|c|c|c|c|c|c|c|}
\hline \multicolumn{10}{|c|}{ SUS Score } \\
\hline \multirow{2}{*}{ Grupo I } & App-A & 100 & 97,5 & 95 & 92,5 & 82,5 & 90 & 70 & 82,5 & - \\
& App-B & 100 & 40 & 80 & 95 & 65 & 92,5 & 80 & 40 & - \\
\hline \multirow{2}{*}{ Grupo II } & App-A & 100 & 97,5 & 100 & 85 & 87,5 & 87,5 & 95 & 80 & 100 \\
& App-B & 27,5 & 55 & 77,5 & 55 & 55 & 37,5 & 80 & 70 & 62,5 \\
\hline
\end{tabular}

A partir da Tabela 3, é possível observar a avaliação de cada participante para ambos os aplicativos. As notas atribuídas pelos usuários são bastante diferentes em relação aos aplicativos por grupo. Esse fato pode ter ocorrido em função de os cenários usados por cada um não serem iguais, sendo assim, ao ver diferentes funções foram atribuídas diferentes notas. Cabe salientar que a união dos cenários contempla completamente as funcionalidades de ambos os aplicativos.

Segundo [Sauro 2011], para que uma aplicação seja considerada com bom nível de usabilidade, o resultado da SUS deve apresentar uma média acima de 68 pontos. Assim, 
além da média final de cada aplicativo, calculada a partir dos resultados dos grupos I e II, a Figura 2 traz a informação da média calculada a partir de uma simulação. Nela, um participante avaliaria da melhor forma os dois aplicativos, já que o grupo I possuía um participante a menos. A simulação foi feita para que fosse possível mostrar que, mesmo com uma avaliação excelente, o resultado final obtido não seria afetado.

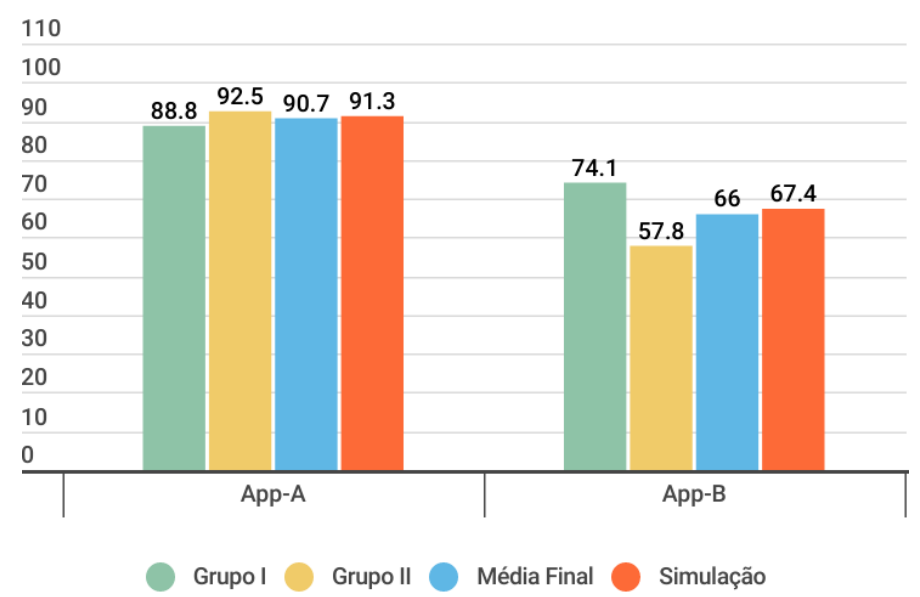

Figura 2. Gráfico da pontuação final SUS.

Sendo assim, pela Figura 2, observa-se que o App-A teve sua pontuação final alterada de 90,7 para 91,3, se mantendo acima da pontuação mínima de qualidade. Da mesma maneira, o App-B, mesmo após a simulação, permaneceu abaixo da pontuação mínima, alterando sua média de 66,0 para 67,4. Buscando evitar que os valores adicionados na simulação influenciassem no resultado final, realizou-se outra simulação, na qual a média final da SUS foi calculada com a ausência das notas de um participante do grupo II por vez. As médias no App-A se mantiveram entre 91,6 e 94,1, permanecendo acima dos 68 pontos mínimos em todas as simulações. Já o aplicativo App-B manteve as médias entre 55,0 e 60,3, permanecendo com notas abaixo da pontuação esperada. A partir da execução das duas simulações, pode-se concluir que a diferença no número de participantes em um dos grupos não afetou diretamente o resultado final obtido pelo estudo.

Para demonstrar a distribuição e amplitude dos resultados obtidos pela SUS, a Figura 3 apresenta um boxplot das notas de cada app.

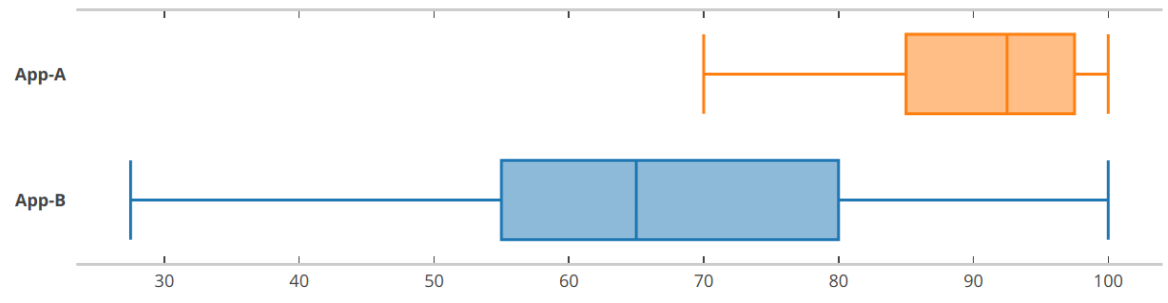

Figura 3. Gráfico Boxplot das notas obtidas pela SUS.

Pela Figura 3 pode-se constatar que, de acordo com os valores atribuídos, o AppA contém uma menor variação nas notas atribuídas pelos participantes. Ainda, observa-se que a mediana referente ao App-B ficou abaixo da outra aplicação, e os valores máximos atribuídos em cada aplicativo são os mesmos, porém o App-B obteve um valor mínimo 
muito menor que o App-A. Todas essas informações, melhor visualizadas no boxplot, confirmam o desempenho melhor do App-A em relação ao App-B.

É possível afirmar que os resultados obtidos com a medida SUS confirmam o que era esperado pelos autores. Em um primeiro uso dos aplicativos, percebeu-se que, embora ambos tenham quase as mesmas funcionalidades, o App-A (Tablas de Verdad PRO) se mostrava mais atrativo, com uma interface mais simples e direta para uso. Por outro lado, vale lembrar que App-B (The Logic Calculator) é mais completo, permitindo avaliação de formas de argumento.

\subsection{Avaliação Dissertativa e Sugestão de Melhorias}

No âmbito qualitativo, o formulário de avaliação continha uma questão dissertativa não obrigatória, na qual os participantes poderiam realizar quaisquer comentários sobre os aplicativos avaliados. Sobre a aplicação App-A, os usuários demonstraram ter tido uma boa experiência, houveram elogios sobre a paleta de cores, interface simples e minimalista, assim como às funcionalidades para suporte ao aprendizado. Como pontos negativos foram citados o idioma do aplicativo (espanhol), o tamanho pequeno dos botões e a ausência de uma funcionalidade para realização de dedução natural. Quanto ao App-B, os participantes se mostraram confusos com o uso da interface, sendo que em uma das opiniões foi descrito que a interface é "complexa e parcialmente incompleta". Também houveram ponderações sobre o idioma, nesse caso o inglês e a organização dos botões da interface.

Junto à avaliação submetida pelos usuários, os autores também notaram aspectos que podem ser melhorados. No aplicativo App-A é indicada a inserção da funcionalidade de verificação da validade de argumentos, assim como a função de copiar tabela para área de transferência. Adicionalmente, sugere-se a inserção de pequenas explicações sobre OL, o que ocorria na versão anterior. Já o App-B poderia realizar melhorias referentes à proximidade dos botões do teclado, assim como no espaçamento da cópia da tabela gerada, além de modificações na interface para torná-la mais atrativa.

\subsection{Ameaças à Validade}

No presente trabalho existem algumas limitações que poderiam influenciar no resultado e, por isso, foram tratadas para que fosse possível minimizá-las. A primeira ameaça encontrada foi a possibilidade de ter no teste participantes que possuam conhecimento da existência dos aplicativos, pois eles teriam uma maior facilidade de uso devido ao conhecimento prévio da ferramenta. Para contornar esta ameaça, selecionou-se participantes que não foram apresentados aos aplicativos durante o ensino dos conteúdos na disciplina. Como segunda ameaça, apontamos o tamanho amostral muito pequeno para uma análise de dados. Para mitigar essa ameaça, foram elaborados dois cenários para cada aplicativo. Assim, utilizou-se o total de participantes para avaliar cada uma das aplicações.

O fato de todos os participantes serem alunos da disciplina de $\mathrm{LM}$ também configura uma ameaça. Como os aplicativos possuem foco educacional, tendo como grupo específico alunos da disciplina de LM, percebeu-se que é possível ampliar a aplicação dos testes com perfis de participantes diferentes (professores, mestrandos), porém a ampliação dos perfis pode não influenciar diretamente o resultado final. Já em relação a aplicação do teste em si, viu-se como ameaça o participante não possuir smartphone com sistema 
operacional Android. Para evitar que ocorresse esse problema, fez-se um aviso prévio sobre o requisito do sistema operacional. Pode-se acrescentar que o fato do teste ter sido dividido em dois grupos e ter ocorrido com moderadores diferentes em cada grupo poderia ser uma ameaça, mas foi mitigada, visto que todas as informações foram passadas com os dois grupos juntos, após, quando os grupos foram separados, nada mais foi dito, apenas foram passados os arquivos de cenário e avaliação dos aplicativos.

\section{Considerações Finais}

Considera-se que o uso de ferramentas TDICs unido aos conteúdos passados em sala de aula possa ser um importante auxílio para que as dificuldades encontradas em LM sejam ultrapassadas. Entretanto, faz-se necessária a avaliação dessas ferramentas para que se possa assegurar entendimento do conteúdo ao utilizar elas. $\mathrm{O}$ teste de usabilidade com os aplicativos App-A e App-B foi realizado com a participação de 17 discentes divididos em 2 grupos, para os quais foram criados 4 cenários, com o objetivo de que todos testassem as 2 aplicações. Após análise da escala SUS foi possível perceber que o aplicativo App-A atingiu média 90,7 classificando-o com um bom nível de usabilidade, enquanto AppB não atingiu a média final mínima. Assim, pode-se concluir que na visão dos alunos participantes do teste, o uso do aplicativo App-A é indicado no ensino de TV, podendo ser incluído como ferramenta de apoio ao ensino e aprendizado de LM.

Apesar dos pontos que podem ser melhorados, o App-A, Tablas de Verdad PRO, teve uma boa avaliação dos usuários devido aos seus pontos fortes destacados anteriormente, como a boa experiência do usuário, a boa paleta de cores e um aplicativo simples e fácil de usar. Diferentemente, o App-B não contém uma interface intuitiva, dificultando o entendimento. Além disso, geração da cópia da TV] resultante necessita uma melhor divisão entre operadores e valores, para ser melhor visualizada. Por fim, de um modo geral, acredita-se que ambos aplicativos podem ser utilizados no intuito de conferir resultados e possíveis erros das resoluções de exercícios. Porém, precisam ser melhorados para contribuírem no ensino e aprendizado dos alunos.

Como trabalhos futuros, pretende-se coletar dados do desempenho dos alunos na disciplina de LM em atividades que envolvam a solução de TV, a partir do uso do aplicativo indicado neste trabalho. Também se tem como objetivo o desenvolvimento de um protótipo de aplicativo, que contemple as funcionalidades do App-B e seja construído com base em requisitos pedagógicos para uso em sala de aula.

\section{Referências}

Andrade, M. V. M., Araújo Jr., C. F., and Silveira, I. F. (2017). Estabelecimento de critérios de qualidade para aplicativos educacionais no contexto dos dispositivos móveis (M-Learning). EaD em Foco, 7(2).

Brooke, J. (1996). SUS: a "quick and dirty" usability. Usability evaluation in industry, 189.

de Souza, J. N. (2008). Lógica para Ciência da Computação: uma introdução concisa. ELSEVIER, 2 edition.

Dias, B. and Finger, A. (2020). Aplicativos para o ensino-aprendizagem de lógica matemática: qual a melhor escolha? In Anais do XXVI Workshop de Informática na Escola, pages 111-120, Porto Alegre, RS, Brasil. SBC. 
Fernandez, A., Insfran, E., and Abrahão, S. (2011). Usability evaluation methods for the web: A systematic mapping study. Information and Software Technology, 53(8):789817. Advances in functional size measurement and effort estimation - Extended best papers.

Gersting, J. L. (2001). Fundamentos Matemáticos para a Ciência da Computação. LTC, 3 edition.

Jurgina, L., Zanandrea, R., Júnior, L. R., and Marques, F. (2020). Logicflow: Uma ferramenta para o auxílio de ensino-aprendizagem de circuitos digitais. In Anais do XXXI Simpósio Brasileiro de Informática na Educação, pages 322-331, Porto Alegre, RS, Brasil. SBC.

Likert, R. (1932). A technique for the measurement of attitudes. Archives of psychology.

Peres, S. C., Pham, T., and Phillips, R. (2013). Validation of the system usability scale (sus): Sus in the wild. Proceedings of the Human Factors and Ergonomics Society Annual Meeting, 57(1):192-196.

Pressman, R. S. (2011). Engenharia de Software: Uma Abordagem Profissional. McGraw-Hill, São Paulo, 7 edition.

Sauro, J. (2011). Measuring Usability With the System Usability Scale (SUS). Disponível em: https://measuringu.com/sus/.

SBC (2018). Educação superior em computação Estatísticas - 2017. Sociedade Brasileira de Computação. Disponível em: https://www.sbc.org.br/documentosda-sbc/summary/133-estatisticas/1200-pdf-png-educacao-superior-em-computacaoestatisticas-2017. Acesso em: 28 fev. 2020.

Sommerville, I. (2011). Engenharia de Software. Pearson Prentice Hall, São Paulo, 9 edition.

Souto, M. and Silva, C. (2017). Um catálogo de requisitos pedagógicos para auxiliar o desenvolvimento de softwares educacionais. Brazilian Symposium on Computers in Education (Simpósio Brasileiro de Informática na Educação - SBIE), 28(1):506.

Souza, M. and França, C. (2016). O que Explica o Sucesso de Jogos no Ensino de Engenharia de Software? Uma Teoria de Motivação. In Anais do XXIV Workshop sobre Educação em Computação, pages 260-269, Porto Alegre, RS, Brasil. SBC.

Valentim, N., Rabelo, J., Silva, W., Coutinho, W., Álvaro Mota, and Conte, T. (2014). Avaliando a qualidade de um aplicativo web móvel através de um teste de usabilidade: um relato de experiência. In Anais do XIII Simpósio Brasileiro de Qualidade de Software, pages 255-262. SBC. 